\author{
Marquette University \\ e-Publications@Marquette
}

Civil and Environmental Engineering Faculty

Civil, Construction, and Environmental

Research and Publications

Engineering, Department of

2013

\title{
Cantilever-based Resonant Gas Sensors with Integrated Recesses for Localized Sensing Layer Deposition
}

C. Carron

Georgia Institute of Technology - Main Campus

P. Getz

Georgia Institute of Technology - Main Campus

J. J. Su

Georgia Institute of Technology - Main Campus

D. S. Gottfried

Georgia Institute of Technology - Main Campus

Fabien Josse

Marquette University, fabien.josse@marquette.edu

See next page for additional authors

Follow this and additional works at: https://epublications.marquette.edu/civengin_fac

Part of the Civil and Environmental Engineering Commons

\section{Recommended Citation}

Carron, C.; Getz, P.; Su, J. J.; Gottfried, D. S.; Josse, Fabien; and Heinrich, Stephen M., "Cantilever-based Resonant Gas Sensors with Integrated Recesses for Localized Sensing Layer Deposition" (2013). Civil and Environmental Engineering Faculty Research and Publications. 22.

https://epublications.marquette.edu/civengin_fac/22 
Authors

C. Carron, P. Getz, J. J. Su, D. S. Gottfried, Fabien Josse, and Stephen M. Heinrich 
Marquette University

e-Publications@Marquette

\section{Civil, Construction and Environmental Engineering Faculty Research and Publications/College of Engineering}

This paper is NOT THE PUBLISHED VERSION.

Access the published version via the link in the citation below.

2013 IEEE Sensors Proceedings, (2013). DOI. This article is (C) Institute of Electrical and Electronic Engineers (IEEE) and permission has been granted for this version to appear in $\underline{\text { e- }}$ Publications@Marquette. Institute of Electrical and Electronic Engineers (IEEE) does not grant permission for this article to be further copied/distributed or hosted elsewhere without the express permission from Institute of Electrical and Electronic Engineers (IEEE).

\section{Cantilever-Based Resonant Gas Sensors with Integrated Recesses for Localized Sensing Layer Deposition}

\section{Carron}

School of Electrical and Computer Engineering, Georgia Institute of Technology, Atlanta, GA P. Getz

School of Electrical and Computer Engineering, Georgia Institute of Technology, Atlanta, GA J.-J. Su

School of Electrical and Computer Engineering, Georgia Institute of Technology, Atlanta, GA D.S. Gottfried Institute for Electronics and Nanotechnology, Georgia Institute of Technology, Atlanta, GA O. Brand School of Electrical and Computer Engineering, Georgia Institute of Technology, Atlanta, GA F. Josse Department of Electrical and Computer Engineering, Marquette University, Milwaukee, WI 


\title{
S. M. Heinrich
}

Department of Civil, Construction and Environmental Engineering, Marquette University, Milwaukee, WI

\begin{abstract}
:
This work presents mass-sensitive hammerhead resonators with integrated recesses as a gas-phase chemical microsensor platform. Recesses are etched into the head region of the resonator to locally deposit chemically sensitive polymers by ink-jet printing. This permits the sensing films to be confined to areas that (a) are most effective in detecting mass loading and (b) are not strained during the in-plane vibrations of the resonator. As a result of the second point, even 5- $\mu \mathrm{m}$ thick polymer coatings on resonators with a 9-12 $\mu \mathrm{m}$ silicon thickness barely affect the $\mathrm{Q}$-factor in air. This translates into higher frequency stability and ultimately higher sensor resolution compared to uniformly coated devices.
\end{abstract}

\section{SECTION I. Introduction}

Current technology trends indicate an ever-increasing demand for chemical and biological sensors. Many current and future applications require such sensors to operate in gas or liquid environments, with reduced power consumption and improved sensitivity to very low concentrations of target analytes in the presence of interfering compounds. Various types of sensor platforms are under development to meet these challenges [1]. Of the many available sensor technologies, mass-sensitive cantilever-based sensors [2], [3] are an attractive technology to explore for several reasons. First, the design and theory of cantilever sensors is well understood, and their fabrication via standard MEMS processing techniques is well established. Second, the interface circuitry necessary for measuring frequency shifts in resonant sensors is mature and well documented [4]. Third, all molecules possess mass, which allows for the potential detection of any target species with a mass-sensitive approach. In practice, however, detection of a specific analyte can be complicated by the presence of chemical interference, and accurate detection of extremely low concentrations is bounded by ambient noise and the sensor's inherent limit of detection (LOD).

All three of these issues - chemical interference, noise, and LOD - can be approached simultaneously through improvements in the chemically-sensitive sorbent layer that is typically applied to the surface of mass-sensitive cantilever-based sensors. For example, by coating arrays of sensors, where each individual sensor is coated with a separate class of sorbent, the response of the array can be interpreted as a multidimensional measurement [5]. This multidimensional approach offers the potential of detecting specific target analytes by characteristic chemical fingerprints across the sensor array even in the presence of interfering compounds. Additionally, careful selection of sorbent materials that are disproportionately sensitive to target analytes can significantly enhance the sensitivity of the sensor, which in turn improves the signal-to-noise ratio and LOD.

However, the addition of a uniformly applied sorbent layer over the entire cantilever structure can dramatically degrade a device's mechanical and electrical characteristics. For example, it has been observed that a uniform sorbent coating can degrade a given sensor's initial, uncoated Q-factor by as much as an order of magnitude when operated in air [6]. Thus, the ability to confine the sorbent layer near the tip of the beam offers the advantage of preserving the desirable mechanical properties of the (silicon) beam in high-strain regions, which in turn preserves the $\mathrm{Q}$-factor. Additionally, localized sorbent polymer deposition allows the sorbent to be deposited in regions where it is most effective in detecting mass loading.

Inkjet printing has been demonstrated as one method of achieving localized polymer deposition [7]. With this approach, the sorbent polymer is typically dissolved in a compatible solvent and subsequently ejected onto the 
substrate through a micro-nozzle via piezoelectric actuation. This versatile technique allows for polymer deposition onto nonplanar substrates, and can print arbitrary feature patterns through the use of software scripting. In practice, however, it can prove difficult to deposit high-quality polymer films with inkjet printing for a variety of reasons. For example, the range of suitable solvents can be limited by incompatibility with the printing nozzle itself. It has also been observed that certain polymer/solvent combinations result in non-uniform films, where the deposited polymer film is considerably thicker near the edges of the film and thinner in the center ("coffee ring" effect). Considering cantilever-based chemical sensors, it would be desirable to leverage the advantages of inkjet printing to deposit different classes of sorbent films onto arrays of sensors, with each sorbent film being uniform and localized near the tip of the cantilever beam, away from high-strain regions near the beam support.

To this end, a novel technique for improving the quality of deposited sorbent films with significantly reduced Qfactor degradation for the resonator operated in air is demonstrated. The technique consists of forming an integrated recess structure into the head region of a silicon hammerhead cantilever designed to resonate in an in-plane flexural mode. The sorbent film is deposited via inkjet printing and is localized in the recess structure, a significant distance away from high-strain areas of the cantilever support beam (Fig. 1).

\section{SECTION II. Design \& Fabrication}

The hammerhead resonators tested in this work consist of a semicircular head portion with inner and outer radii of $100-\mu \mathrm{m}$ and $200-\mu \mathrm{m}$, respectively, supported by a $45-\mu \mathrm{m}$ wide and $100-\mu \mathrm{m}$ long cantilever beam. At its supported end, each hammerhead structure has embedded silicon resistors for electrothermal excitation and piezoresistive detection of in-plane flexural vibrations [8]. The fabrication process for the cantilever-based microstructures is highlighted in [9]. The hammerhead sensors exhibit relatively low Q-factors for out-of-plane modes, due to increased viscous damping from the large surface area of the head region. Operating the devices in an in-plane flexural mode, however, enhances the Q-factor as the beam effectively slices through the surrounding medium, experiencing significantly reduced energy loss from viscous damping despite its large surface area in the plane. Exciting the hammerhead sensors in an in-plane mode allows even liquid-phase operation with reasonably high Q-factors [10].

Using reactive ion etching, an approximately $5-\mu$ m deep recess was etched into the head area of the silicon hammerhead, the latter having a nominal thickness of $9-12 \mu \mathrm{m}$ (Fig. 1 ). This recess actually slightly reduces the $\mathrm{Q}$-factor of the in-plane mode as the resonator mass is decreased while keeping surface losses to the surrounding fluid approximately the same. Prior to mounting the chip containing eight recessed resonators into a dual-in-line package, ink-jet printing (Microfab JetLab II) was used to fill the recess of select resonators with a sorbent polymer layer. Each resonator was coated individually with only one type of sorbent polymer - in the present work, either polyvinylacetate (PVAc) or polyisobutylene (PIB) - which serves as the chemically sensitive film. To avoid nozzle clogging, dimethyl sulfoxide was used as a solvent for PVAc, and xylene as a solvent for PIB, because of their relatively high boiling points and thus slow rate of evaporation during the ink-jetting process. Various parameters such as solvent to polymer mass ratio, jet nozzle orifice diameter, and the jet nozzle's piezoelectric voltage levels and timing influence the accuracy and quality of the deposited films. Bursts of drops were deposited at various locations in the recess, allowed to evaporate, and followed by another burst until the recess was completely filled. To counter the high boiling point of the solvents and allow for more frequent bursts, the JetLab stage was heated. In this way the recesses could be completely filled with polymer (Fig. 1) without covering any of the support beam. Moreover, the recess reduces solvent splattering and nicely confines the deposited polymer. Examination of the images in Fig. 1, but also analysis of the resonance frequency before and after coating, indicate that the recess structure is filled with approximately $3-\mu \mathrm{g}$ of PVAc. 

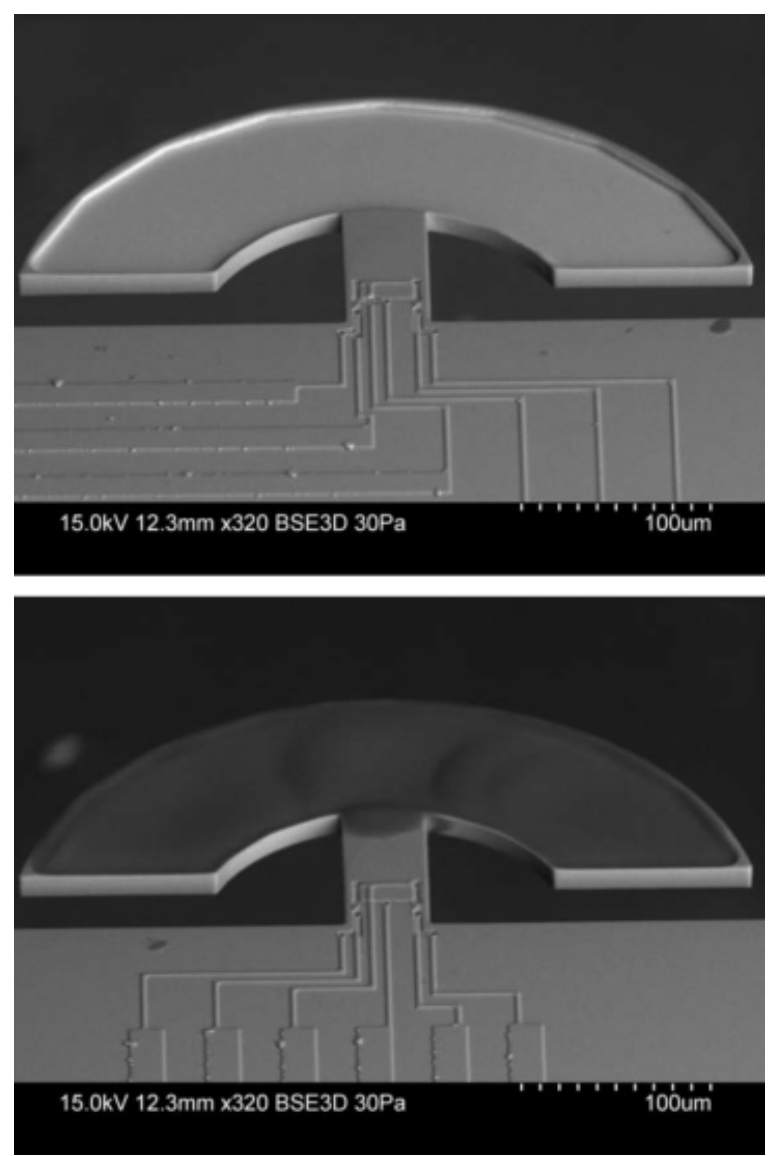

Figure 1. SEM micrographs of (a) uncoated hammerhead resonator with $12-\mu \mathrm{m}$ total thickness and recess of $5-\mu \mathrm{m}$ etched into the head structure and (b) hammerhead resonator after filling the recess with polyvinylacetate (PVAc) as localized chemically sensitive film via ink-jet printing

It is evident from Fig. 1 that the integrated recess structure functions well in containing the deposited polymer into a smooth, uniform, and localized film near the beam tip. In addition to mitigating the coffee ring effect and improving the uniformity of the sorbent layer, the sorbent-filled recess structure enhances overall device sensitivity by effectively replacing silicon with sorbent layer "active mass" that is chemically sensitive. Thus, a hammerhead sensor with integrated recess is inherently more sensitive, for a given cantilever and polymer thickness, than a non-recessed device.

The effect of the integrated recess structure on the device performance was initially modeled using finite element analysis (COMSOL Multiphysics). Simulation indicated that the introduction of a $5-\mu \mathrm{m}$ integrated recess structure into an uncoated $9-\mu \mathrm{m}$ thick hammerhead device resulted in an expected fundamental inplane resonance frequency of $489 \mathrm{kHz}$ for the hammerhead device (Figure 2). Simulation further showed that the localized deposition of $5-\mu \mathrm{m}$ of PIB into the recess structure resulted in a resonant frequency drop to 450 $\mathrm{kHz}$ due to the added mass of the sorbent polymer layer. The simulations were found to be in close agreement with experimental data: the recessed $9-\mu \mathrm{m}$ hammerhead structure exhibited a measured in-plane resonance frequency of approximately $483 \mathrm{kHz}$ prior to coating. Localized deposition of $5-\mu \mathrm{m}$ of PIB via inkjet printing lowered the resonance frequency to $445 \mathrm{kHz}$. In case of the device coated with $7-\mu \mathrm{m}$ PVAc, the simulation yielded an in-plane resonance frequency of $420 \mathrm{kHz}$, while the measured frequency was $410 \mathrm{kHz}$ (see Fig. 3). 


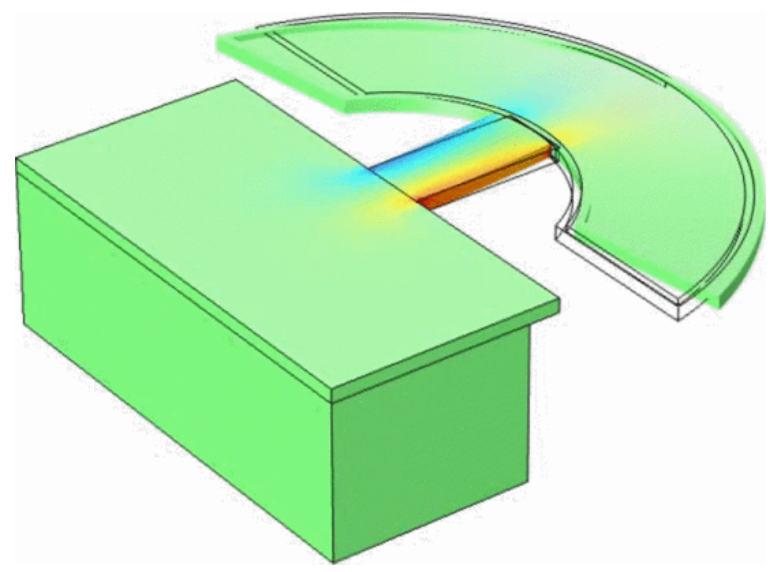

Figure 2. Finite element modal analysis of $9-\mu \mathrm{m}$ thick hammerhead resonator with $5-\mu \mathrm{m}$ recess. The figure shows the stress in $\mathrm{x}$-direction at the fundamental in-plane mode ( $\mathrm{f}=489 \mathrm{kHz})$; blue represents regions under compressive stress, red regions under tensile stress, while green indicates "stress-free" regions

The finite element simulations also confirm that the recess is confining the polymer to areas away from the strained region of the microstructure (see stress distribution in Fig. 2). Finally, the sensitivity of the device to changes in the polymer density (i.e., the change in frequency per change in polymer density [11]) was explored: a $9-\mu \mathrm{m}$ thick hammerhead without recess coated with a uniform $5-\mu \mathrm{m}$ PIB film exhibits a simulated sensitivity of $36 \mathrm{~Hz} /\left(\mathrm{kgm}^{-3}\right)$, while the $9-\mu \mathrm{m}$ hammerhead with a $5-\mu \mathrm{m}$ recess filled completely with PIB shows a sensitivity of $46 \mathrm{~Hz} /\left(\mathrm{kgm}^{-3}\right)$. Even though not all surface area is coated with polymer, the fact that silicon is locally replaced by polymer improves the gravimetric sensitivity of the recessed device. At the same time, the Q-factor in air is improved by the localized polymer deposition and, both, the sensitivity and Q-factor improvements are expected to yield better limits of detection in a chemical sensing application.

\section{SECTION III. Experimental Results}

\section{A. Resonance Frequency and Quality Factor}

The transfer characteristics of uncoated recessed hammerhead devices were compared to those of recessed devices with ink-jet printed coatings (Fig. 3), and to those of non-recessed devices with spray-coated sorbent layers. Previous work has shown that uniform deposition of a $7-\mu \mathrm{m}$ sorbent film over an entire resonator structure (including the highly strained regions near the support) can lead to Q-factor degradation of one order of magnitude due to damping induced by periodically deforming the polymer [6]. Through application of the integrated recess structure with a localized polymer deposition, the Q-factor degradation of the coated devices was significantly reduced. For example, a localized $5-\mu \mathrm{m}$ PIB film resulted in a Q-factor decrease of $\sim 40 \%$, and a $7-\mu \mathrm{m}$ film of PVAc resulted in a Q-factor drop of even only $\sim 1.5 \%$. These data confirm that confining polymer deposition away from the high-strain areas of the support beam enables relatively thick sorbent layers to be applied to a device while still maintaining a high Q-factor in air. This improvement in post-coating Q-factor, while simultaneously allowing the application of relatively thick sorbent films, leads to enhanced frequency stability and sensor sensitivity when compared to uniformly coated devices. Ultimately, theses factors combine to improve the sensor LOD. 

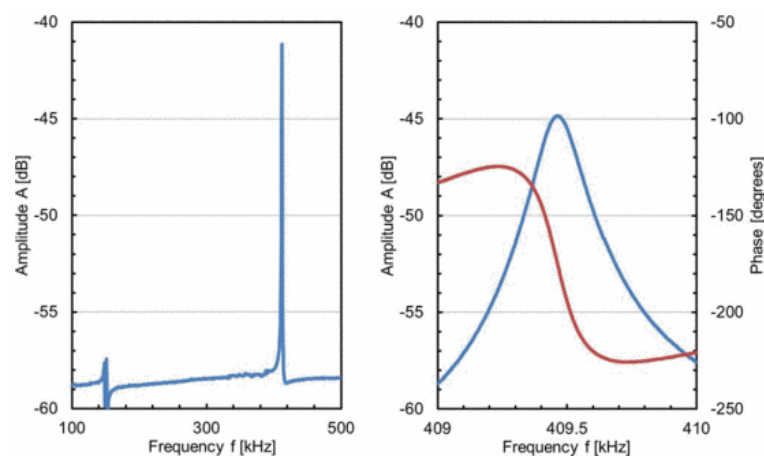

Figure 3. Piezoresistive output signal as a function of excitation frequency of recessed hammerhead device, coated with $\sim 7 \mu \mathrm{m}$ PVAc via ink-jet printing: (left) frequency range from 100-500 kHz showing two flexural modes with the desired in-plane mode at $410 \mathrm{kHz}$ having the strongest signal; (right) close-up of amplitude and phase transfer characteristic of in-plane mode with $\mathcal{Q}=1910$

\section{B. Chemical Measurements}

Following the open-loop characterization, the sorbent-coated sensors were evaluated as chemical sensors. To this end, coated resonators were embedded into an amplifying feedback loop and exposed alternately to pure nitrogen carrier gas and defined concentrations of volatile organic compounds (VOCs) in a custom gas set-up. As an example, Fig. 4 shows the frequency response of a PIB-coated resonator to different concentrations of toluene. The data were collected at a constant temperature of $19^{\circ} \mathrm{C}$ and a flow rate of $80 \mathrm{ml} / \mathrm{min}$ through the measurement chamber. Between successive exposures to the analyte-loaded gas stream, the gas flow over the resonator is changed to pure nitrogen carrier gas. A four-way valve enables fast switching and allows the investigation of signal transients as well. From the baseline frequency data (see final 500 seconds in Fig. 4), a short-term frequency stability of $2 \times 10^{-8}$ was extracted using the Allan variance method.

An analysis of the measured frequency data reveals that the sensors exhibit a linear response with respect to toluene concentration (Fig. 5). Using the observed chemical sensitivity of $0.15 \mathrm{~Hz} / \mathrm{ppm}$ for toluene and the Allan variance of $2 \times 10^{-8}$, limits of detection below $1 \mathrm{ppm}$ can be expected for these devices. However, these LOD values need to be confirmed by measurements at ppm-level analyte concentrations. Another important observation from the gas measurement data is that the sensor's response is fully reversible with time constants well below 1 minute even for relatively thick polymer films.

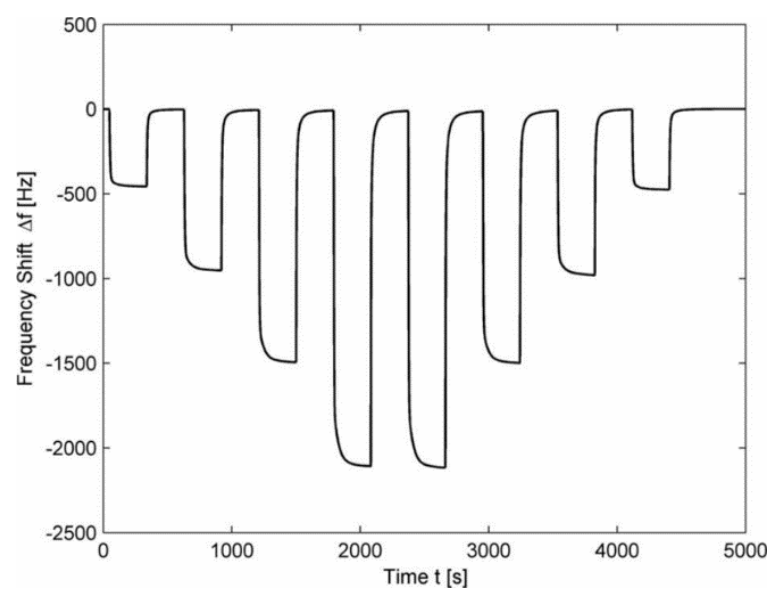

Figure 4. Experimentally observed frequency shift of PIB-coated recessed resonator as a function of time; the microsensor is subsequently exposed to different toluene concentrations (3400-6800-10200-13600-1360010200-6800-3400 ppm). Between successive toluene exposures, the chamber is flushed with nitrogen as carrier gas. A linear drift of $0.3 \mathrm{~Hz} / \mathrm{min}$ has been subtracted from the data 


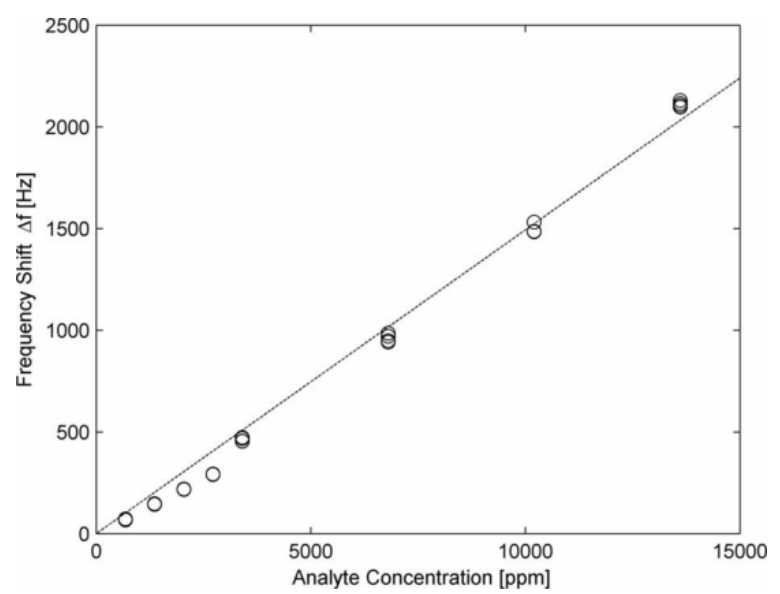

Figure 5. Experimentally observed frequency shift for PIB-coated recessed resonator as a function of the toluene concentration

\section{SECTION IV. Conclusion}

The design, simulation, and characterization of a mass-sensitive chemical sensor platform with integrated recess regions for the localized deposition of sorbent polymer layers is presented. Ink-jet printing offers the potential of localized polymer deposition, but can encounter difficulties with printing onto planar substrates due to, e.g., the "coffee ring" effect. The integrated recesses presented here offer the ability to confine the sorbent layer to a location away from the high-strain regions of the support beam, which holds distinct advantages over uniformlycoated devices in terms of Q-factor degradation, and ultimately translates into enhanced chemical sensitivity and resolution. This is demonstrated through the use of the resonator as a chemical sensor targeting VOCs such as toluene in the gas phase. Future work will examine the possibility of coating and operating arrays of sensors, each with a unique sorbent layer with the goal of reliably detecting target analytes in gas or liquid-phase in the presence of chemical interference.

\section{ACKNOWLEDGMENT}

This work was performed in part at the Georgia Tech Institute for Electronics and Nanotechnology, a member of the National Nanotechnology Infrastructure Network (NNIN), which is supported by the National Science Foundation. The authors would like to thank the Institute for Electronics and Nanotechnology (IEN) for help and support with process development and fabrication.

\section{References}

1. J. Janata, M. Josowicz, P. Vanysek, and D. M. DeVaney, "Chemical sensors," Analytical Chemistry, vol. 70, pp. 179-208, 1998.

2. K.M. Goeders, J.S. Colton, and L.A. Bottomley, "Microcantilevers: Sensing chemical interactions via mechanical motion," Chemical Reviews, vol. 108, pp. 522-542, 2008.

3. N. V. Lavrik, M. J. Sepaniak, and P. G. Datkos, "Cantilever transducers as a platform for chemical and biochemical sensing," Review of Scientific Instruments, vol. 75, pp. 2229-2253, 2004.

4. K.S. Demirci, L.A. Beardslee, S. Truax, J.-J. Su, and O. Brand, "Integrated silicon-based chemical microsystem for portable sensing applications," Sensors and Actuators B, vol. 180, pp. 50-59, 2013.

5. A. Hierlemann and R. Gutierrez-Osuna, "Higher-order chemical sensing," Chem. Rev., vol. 108, pp. 563-613, 2008.

6. S.B. Truax, K.S. Demirci, L.A. Beardslee. Y. Luzinova, A. Hierlemann, B. Mizaikoff, and O. Brand, "Mass-sensitive detection of gas-phase volatile organics using disk microresonators," Analytical Chemistry, vol. 83, pp. 3305-3311, 2011. 
7. H. Kang, D. Soltman, and V. Subramanian, "Hydrostatic optimization of inkjet-printed films," Langmuir, vol. 26, pp. 11568-11573, 2010.

8. L. A. Beardslee, S. M. Heinrich, F. Josse, and O. Brand, "Thermal Excitation and Piezoresistive Detection of Cantilever In-Plane Resonance Modes for Sensing Applications," Journal of Microelectromechanical Systems, vol. 19, pp. 1015-1017, 2010.

9. J. H. Seo and O. Brand, "High Q-factor in-plane mode resonant mircrosensor platform for gaseous/liquid environment," Journal of Microelectromechanical Systems, vol. 17, pp. 483-493, Apr 2008.

10. L.A. Beardslee, S. Aravamudhan, C. Carron, P. Joseph, S.M. Heinrich, F. Josse, and O. Brand, "Detection of anti-IgG using cantilever-type resonant microstructures vibrating in in-plane flexural modes," in Proceedings IEEE Sensors Conference, 2012, pp. 1676-1679.

11. D. Lange, C. Hagleitner, A. Hierlemann, O. Brand, and H. Baltes, "Complementary metal oxide semiconductor cantilever arrays on a single chip: mass-sensitive detection of volatile organic compounds," Analytical Chemistry, vol. 74, pp. 3084-3095, 2002. 